\title{
Potential of core-collapse supernova neutrino detection at JUNO
}

\section{Xin Huang ${ }^{a, *}$ on behalf of the JUNO Collaboration}

(a complete list of authors can be found at the end of the proceedings)

${ }^{a}$ Institute of High Energy Physics, Chinese Academy of Science, Beijing, China

E-mail: huangxin@ihep.ac.cn

JUNO is an underground neutrino observatory under construction in Jiangmen, China. It uses 20kton liquid scintillator as target, which enables it to detect supernova burst neutrinos of a large statistics for the next galactic core-collapse supernova (CCSN) and also pre-supernova neutrinos from the nearby CCSN progenitors. All flavors of supernova burst neutrinos can be detected by JUNO via several interaction channels, including inverse beta decay, elastic scattering on electron and proton, interactions on ${ }^{12} \mathrm{C}$ nuclei, etc. This retains the possibility for JUNO to reconstruct the energy spectra of supernova burst neutrinos of all flavors. The real time monitoring systems based on FPGA and DAQ are under development in JUNO, which allow prompt alert and trigger-less data acquisition of CCSN events. The alert performances of both monitoring systems have been thoroughly studied using simulations. Moreover, once a CCSN is tagged, the system can give fast characterizations, such as directionality and light curve. This talk gives an overview of physics potential of CCSN neutrino detection in JUNO.

$37^{\text {th }}$ International Cosmic Ray Conference (ICRC 2021)

July 12 th - 23rd, 2021

Online - Berlin, Germany

\footnotetext{
*Presenter
} 


\section{Introduction}

At the end of a massive star's whole life, it is believed to be able to collapse and explode to be a core-collapse supernova (CCSN). A CCSN can bring us various detectable signals, including electromagnetic signals, grativational waves and neutrinos. These will help us with the understanding of explosion mechanisms. So it is important to be prepared to observe these signals. As is described in [1], neutrino emission starts before core-collapse, which is called pre-supernova (pre-SN) neutrinos. The pre-SN neutrino emission starts as early as $10^{11} \mathrm{~s}$ before core-collapse and its luminosity increases as time approaches collapse. The SN neutrinos and gravitational waves usually lasts $O(10)$ s, while the shock break out of electromagnetic signals are delayed by $10^{5} \mathrm{~s}$. Also, the time information provided by CCSN neutrinos can improve the sensitivity of gracitational wave detection significantly. Hence, neutrino detection of both pre-SN neutrinos and SN neutrinos can serve as early warnings for CCSN.

Jiangmen Underground Neutrino Observatory (JUNO) [2], located in Jiangmen, China, is a multi-purpose neutrino experiment under construction. The main goal is to measure the neutrino mass ordering. It is designed to be a 20 kton liquid scintillator (LS) detector with 30 kton shielding water and will be the largest LS detetor in the near future. JUNO is equipped with about 18000 20-inch photomultiplier tubes (PMT) and 26000 3-inch PMTs. It is expected to reach 3\%@1MeV energy resolution. Fig. 1 shows a schematic overview of the JUNO detector, which is taken from [3]. JUNO can detect CCSN neutrinos and provide early warning for CCSN.

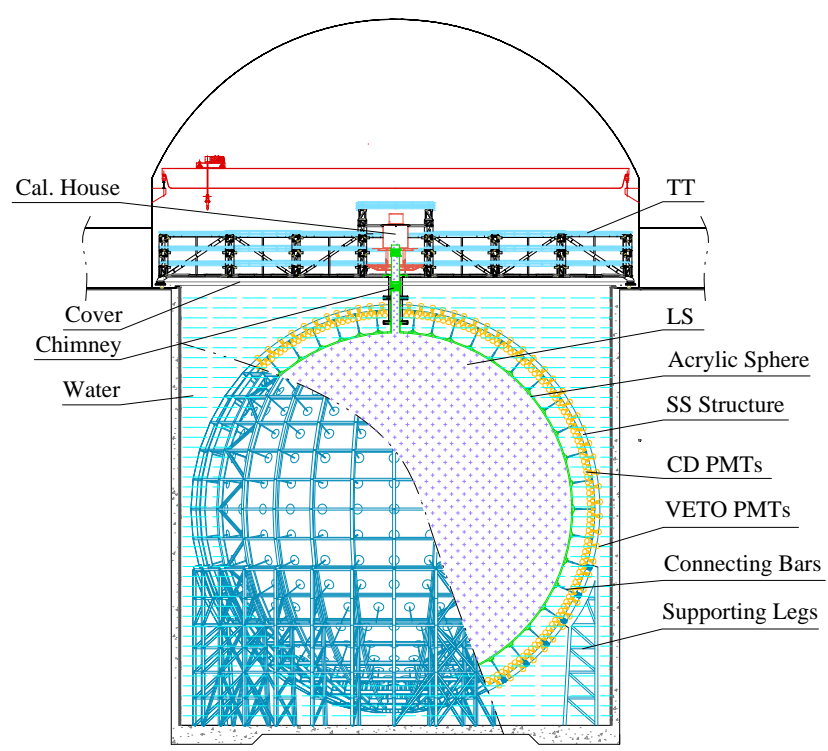

Figure 1: The schematic overview of JUNO detector. 


\section{Supernova neutrino detection at JUNO}

Detecting supernova neutrino is also an important purpose of JUNO. It can see all flavors of neutrinos via several interactions. The main channels are inverse beta decay (IBD), $\bar{v}_{e}+p \rightarrow e^{+}+n$, elastic scattering on electron (eES), $v+e^{-} \rightarrow v+e^{-}$and elastic scattering on proton (pES), $v+p \rightarrow v+p$. JUNO is estimated to detect about 5000 IBDs, 300 eESs and 2000 pESs for CCSN at $10 \mathrm{kpc}$. Beyond these three main detection channels, JUNO can also detect supernova neutrinos through CC and NC interactions with ${ }^{12} C$ nucleis (about 200 events and 300 events @ 10 kpc respectively). The visible energy spectra of all the flavors are shown in Fig. 2 [3].

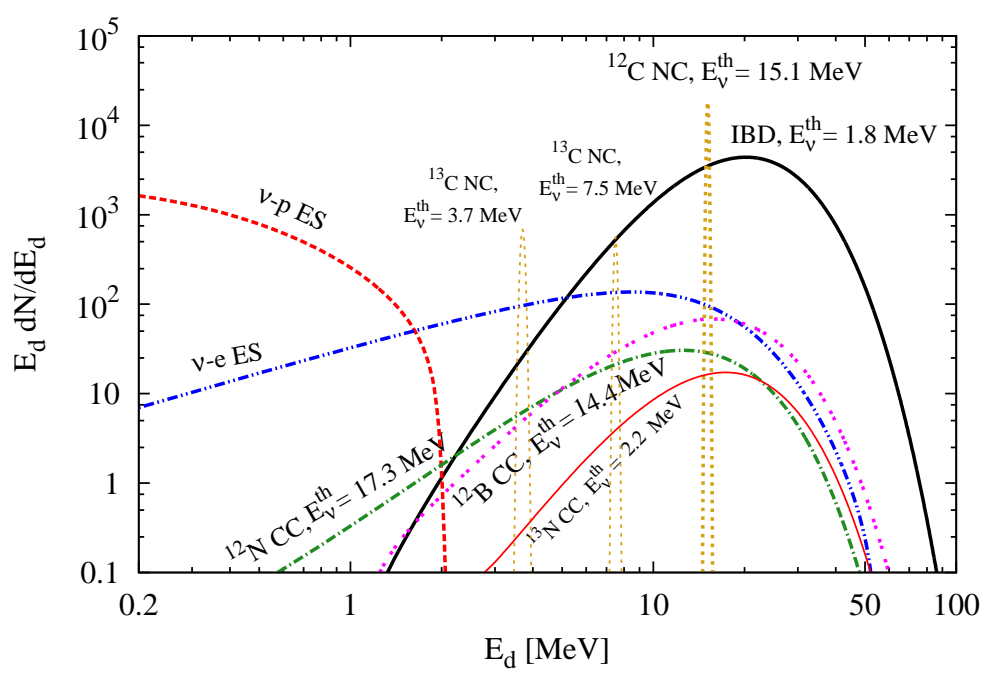

Figure 2: The visible energy spectra at JUNO detector for a typical $\mathrm{SN}$ at $10 \mathrm{kpc}$, where no neutrino flavor conversions are assumed and the average neutrino energies are $\left\langle E_{v_{e}}\right\rangle=12 \mathrm{MeV},\left\langle E_{\bar{v}_{e}}\right\rangle=14 \mathrm{MeV}$ and $\left\langle E_{v_{x}}\right\rangle=16 \mathrm{MeV}$.

\section{Real-time supernova monitor systems at JUNO}

Since when a CCSN happens, the signal rate will increase significantly, we can monitor whether a CCSN happens by monitoring the change of event rate. To provide early alerts for the next CCSN and record CCSN data as much as possible, a real-time CCSN monitor system is designed in JUNO. It is a redundancy design consisting of prompt monitor and online monitor. As is shown in Fig. 3, the prompt monitor is embedded in electronic trigger board which will have short time delay and can hence give fast alert on CCSN, while the online monitor is at the data acquisition (DAQ) stage which utilizes reconstructed information to maximize the monitoring ability. Once an alert is given, the internal collaborators and astronomical communities will be informed.

\subsection{Prompt monitor}

The prompt monitor is made up of two parts based on global trigger and multi-messenger (MM) trigger systems. 


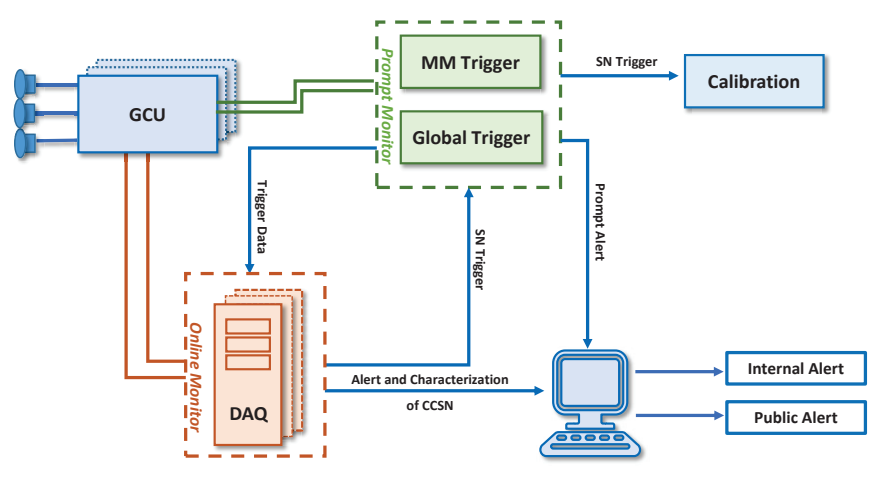

Figure 3: The design of JUNO CCSN monitor systems.

- Based on global trigger: The global trigger system is used to suppress the background events due to PMT dark noise coincidence and radioactivity of ${ }^{14} C$. It has an energy threshold of about $0.2 \mathrm{MeV}$. The monitor system based on global trigger is designed to monitor the rate of supernova neutrino candidates whose energy ranges from about $8 \mathrm{MeV}$ to about $40 \mathrm{MeV}$. It is on the same electronic board with global trigger. Based on the global triggered events, a quantity called $N_{h i t}$, which is approximately proportional to energy, is used to select SN neutrino candidates. $N_{\text {hit }}$ is calculated by counting the total number of pulses of all the PMTs. Fig. 4 shows the detector response of $N_{\text {hit }}$ and the distribution of it from different sources. The dashed lines correspond to the selection criteria of SN candidates. As the figure shows, muons contribute a lot to the background. Hence, the water pool trigger available at the same electronic board as global trigger is applied to veto muon and its related backgrounds. It can reduce the background rate significantly.

- Based on MM trigger: MM trigger system is designed for low energy events. It will reduce the energy threshold to about $20 \mathrm{keV}$. To achieve this, fast filtering algorithms on FPGA is developed to reject dark noise. In the prompt monitor based on MM trigger, signals from all three main channels in JUNO are selected using techniques including pulse shape discrimination (PSD) to distinguish between proton and beta/gamma, electron and positron. Then the event rate can be monitored via algorithms such as Bayesian blocks.

Once an alert is found by prompt monitor system, it will be informed to DAQ and calibration system. The trigger-less T/Q data will be stored by DAQ and the calibration will be suspended.

\subsection{Online monitor}

The online monitor system at DAQ stage implemented in software utilize the reconstructed information to select candidates as in offline analysis. It has the potential to monitor pre-supernova neutrinos, so SN neutrinos and pre-SN neutrinos are monitored seperately. Online monitor uses the trigger-less T/Q data stream in DAQ, and a software trigger is performed to build events from the 


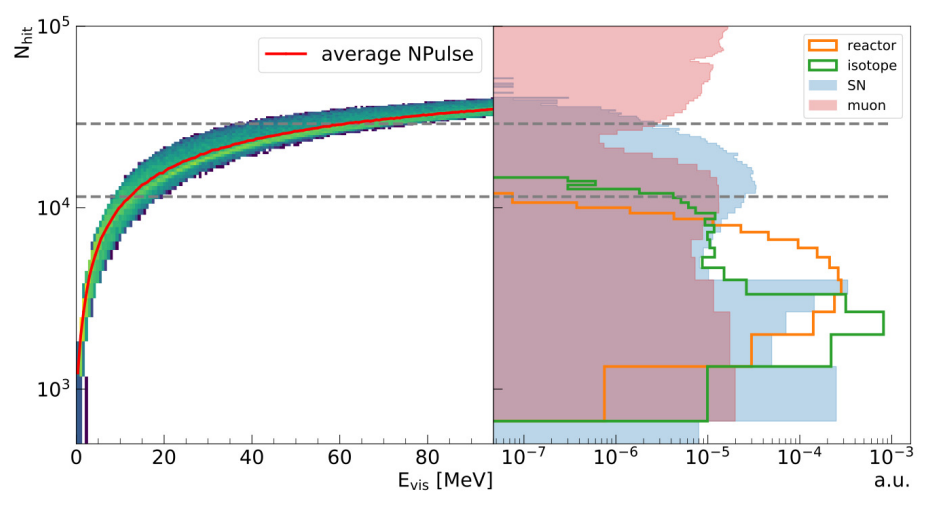

Figure 4: Left: the number of hits $N_{\text {hit }}$ versus the visible energy for a global-triggered event. The red line is the average number of PMT hits with respect to visible energy. Positron samples with energy uniformly distributed from $0 \mathrm{MeV}$ to $100 \mathrm{MeV}$ is used. Right: the distribution of $N_{\text {hit }}$ for different sources, including supernova neutrino, muon, reactor IBD and cosmogenic isotopes. The dashed lines incidate the criteria that are used for event selection.

trigger-less T/Q data. The vertex and energy information are extracted by reconstruction algorithms and used to select IBD events as SN candidates and pre-SN candidates. These candidates are monitored separately by SN monitor and pre-SN monitor. Three types of alert status are defined. An SN alert indicates an alert only from SN monitor, a pre-SN alert indicates an alert only from pre-SN monitor and a nearby alert indicates an alert from pre-SN monitor followed by an alert from $\mathrm{SN}$ monitor within 5 days.

Moreover, in order for fast characterization of CCSN, e.g direction, both SN and pre-SN candidates are stored for one week in the Event Accumulator. Once online monitor finds an alert, the direction of CCSN can be reconstructed using IBDs stored in the Event Accumulator using the formula: $\widehat{D}=\frac{1}{N} \sum_{i} \hat{X}_{p n}^{(i)}$, where $\hat{X}_{p n}^{(i)}$ is the unit vector between the vertex of positron and neutron [4]. JUNO may be able to detect $\mathrm{O}(100)$ pre-SN IBDs for a nearby pre-SN and hence has the potential to give pre-SN direction. For example, Ref. [5] shows JUNO has the potential to reconstruct the direction of pre-SN with the uncertainty of the reconstructed direction to be $70^{\circ}$ at $68 \%$ confidence level for pre-SN at $0.2 \mathrm{kpc}$ (with about 650 IBDs).

\section{Energy spectrum unfolding}

Through three main interaction channels, JUNO can detect all flavors of neutrinos and hence has the potential to reconstruct the energy spectra of all flavors. Here, works from [6] is used as an example for illustration. In this work, a model independent approach is proposed to extract the energy spectra of all flavors. The relationship between observed spectra of three main channels IBD, eES and pES and the flux of all flaovrs $\bar{v}_{e}, v_{e}$ and $v_{x}$ ( $x$ stands for $\mu$ and $\tau$ tyes) can be modeled simply by: $A x=b$, where $A$ is the response matrix reflecting the detector response and interaction cross section, $x$ is the flux of three flaovrs and $b$ is the observed spectra. In this linear form, unfolding method can be performed to extract the spectra of all flavors. Fig. 5 shows the unfolding result for SN at $10 \mathrm{kpc}$. The flux of $\bar{v}_{e}$ is mainly extracted from IBD events while the eES 
and $\mathrm{pES}$ provide the information for $v_{e}$ and $v_{x}$. Note the unfolded flux of $v_{e}$ and $v_{x}$ at low energy is bad. This is due to the $0.2 \mathrm{MeV}$ energy threshold at JUNO, which blinds the information from pES channel at low energy.

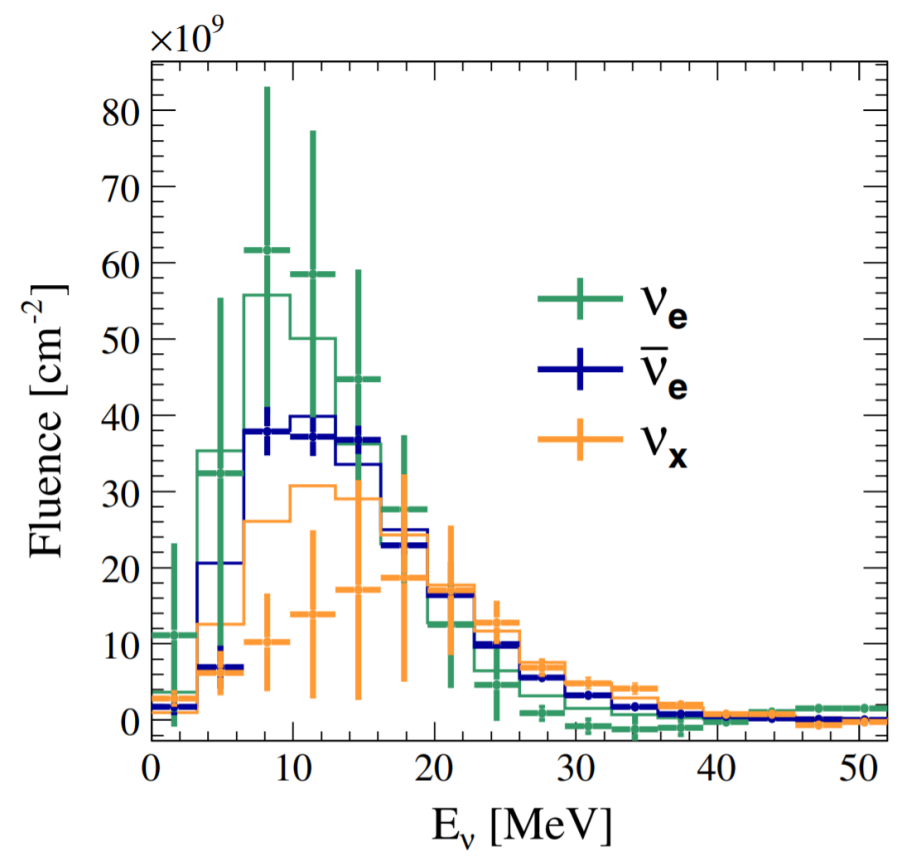

Figure 5: The unfolded energy spectra for a typical SN at $10 \mathrm{kpc}$, taken from [6] as an example.

\section{Summary}

As the largest LS detector in the near future, JUNO can detect all flaovrs of neutrinos through several interaction channels, which enables the potential for JUNO to provide spectral information of all flavors. To give alert to CCSN and store CCSN related data, a redundancy design of CCSN monitor systeim at JUNO is proposed which consists of both prompt monitor and online monitor. The prompt monitor can be based on global trigger or MM trigger to give fast alert on SN, while the online monitor utilizes reconstructed information to select IBD events both for SN and pre-SN. Also, the accumulated events in online monitor are able to give the direction of CCSN, both from SN IBDs and pre-SN IBDs.

\section{References}

[1] K. Nakamura, S. Horiuchi, M. Tanaka, K. Hayama, T. Takiwaki and K. Kotake, "Multimessenger signals of long-term core-collapse supernova simulations: synergetic observation strategies,” Mon. Not. Roy. Astron. Soc. 461 (2016) no.3, 3296-3313

[2] F. An et al. [JUNO], “Neutrino Physics with JUNO,” J. Phys. G 43 (2016) no.3, 030401

[3] A. Abusleme et al. [JUNO], "JUNO Physics and Detector," [arXiv:2104.02565 [hep-ex]]. 
[4] M. Mukhopadhyay, C. Lunardini, F. X. Timmes and K. Zuber, "Presupernova neutrinos: directional sensitivity and prospects for progenitor identification," Astrophys. J. 899 (2020) no. 2,153

[5] H. L. Li, Y. F. Li, L. J. Wen and S. Zhou, "Prospects for Pre-supernova Neutrino Observation in Future Large Liquid-scintillator Detectors," JCAP 05 (2020), 049

[6] H. L. Li, X. Huang, Y. F. Li, L. J. Wen and S. Zhou, "Model-independent approach to the reconstruction of multiflavor supernova neutrino energy spectra," Phys. Rev. D 99 (2019) no.12, 123009 


\section{Full Authors List: JUNO Collaboration}

Angel Abusleme ${ }^{5}$, Thomas Adam ${ }^{45}$, Shakeel Ahmad ${ }^{66}$, Rizwan Ahmed ${ }^{66}$, Sebastiano Aiello ${ }^{55}$, Muhammad Akram ${ }^{66}$, Fengpeng $\mathrm{An}^{29}$, Qi $\mathrm{An}^{22}$, Giuseppe Andronico ${ }^{55}$, Nikolay Anfimov ${ }^{67}$, Vito Antonelli ${ }^{57}$, Tatiana Antoshkina ${ }^{67}$, Burin Asavapibhop ${ }^{71}$, João Pedro Athayde Marcondes de Andre $^{45}$, Didier Auguste ${ }^{43}$, Andrej Babic ${ }^{70}$, Nikita Balashov ${ }^{67}$, Wander Baldini ${ }^{56}$, Andrea Barresi ${ }^{58}$, Davide Basilico ${ }^{57}$, Eric Baussan ${ }^{45}$, Marco Bellato ${ }^{60}$, Antonio Bergnoli ${ }^{60}$, Thilo Birkenfeld ${ }^{48}$, Sylvie Blin ${ }^{43}$, David Blum ${ }^{54}$, Simon Blyth ${ }^{40}$, Anastasia Bolshakova ${ }^{67}$, Mathieu Bongrand ${ }^{47}$, Clément Bordereau ${ }^{44,40}$, Dominique Breton ${ }^{43}$, Augusto Brigatti ${ }^{57}$, Riccardo Brugnera ${ }^{61}$, Riccardo Bruno $^{55}$, Antonio Budano ${ }^{64}$, Mario Buscemi ${ }^{55}$, Jose Busto ${ }^{46}$, Ilya Butorov ${ }^{67}$, Anatael Cabrera ${ }^{43}$, Hao Cai ${ }^{34}$, Xiao Cai ${ }^{10}$, Yanke $\mathrm{Cai}^{10}$, Zhiyan Cai ${ }^{10}$, Riccardo Callegari ${ }^{61}$, Antonio Cammi ${ }^{59}$, Agustin Campeny ${ }^{5}$, Chuanya Cao ${ }^{10}$, Guofu $\mathrm{Cao}^{10}{ }^{1} \mathrm{Jun} \mathrm{Cao}^{10}$, Rossella Caruso ${ }^{55}$, Cédric Cerna ${ }^{44}$, Jinfan Chang ${ }^{10}$, Yun Chang ${ }^{39}$, Pingping Chen ${ }^{18}$, Po-An Chen ${ }^{40}$, Shaomin Chen ${ }^{13}$, Xurong Chen ${ }^{26}$, Yi-Wen Chen ${ }^{38}$, Yixue Chen ${ }^{11}$, Yu Chen ${ }^{20}$, Zhang Chen ${ }^{10}$, Jie Cheng ${ }^{10}$, Yaping Cheng ${ }^{7}$, Alexey Chetverikov $^{67}$, Davide Chiesa ${ }^{58}$, Pietro Chimenti ${ }^{3}$, Artem Chukanov ${ }^{67}$, Gérard Claverie ${ }^{44}$, Catia Clementi ${ }^{62}$, Barbara Clerbaux ${ }^{2}$, Selma Conforti Di Lorenzo ${ }^{44}$, Daniele Corti ${ }^{60}$, Flavio Dal Corso $^{60}$, Olivia Dalager ${ }^{74}$, Christophe De La Taille ${ }^{44}$, Jiawei Deng ${ }^{34}$, Zhi Deng ${ }^{13}$, Ziyan Deng ${ }^{10}$, Wilfried Depnering ${ }^{52}$, Marco Diaz ${ }^{5}$, Xuefeng Ding ${ }^{57}$, Yayun Ding ${ }^{10}$, Bayu Dirgantara ${ }^{73}$, Sergey Dmitrievsky $^{67}$, Tadeas Dohnal ${ }^{41}$, Dmitry Dolzhikov ${ }^{67}$, Georgy Donchenko ${ }^{69}$, Jianmeng Dong ${ }^{13}$, Evgeny Doroshkevich ${ }^{68}$, Marcos Dracos ${ }^{45}$, Frédéric Druillole ${ }^{44}$, Ran Du ${ }^{10}$, Shuxian $\mathrm{Du}^{37}$, Stefano Dusini $^{60}$, Martin Dvorak ${ }^{41}$, Timo Enqvist ${ }^{42}$, Heike Enzmann ${ }^{52}$, Andrea Fabbri ${ }^{64}$, Lukas Fajt ${ }^{70}$, Donghua Fan ${ }^{24}$, Lei Fan ${ }^{10}$, Jian Fang ${ }^{10}$, Wenxing Fang ${ }^{10}$, Marco Fargetta ${ }^{55}$, Dmitry Fedoseev ${ }^{67}$, Vladko Fekete ${ }^{70}$, Li-Cheng Feng ${ }^{38}$, Qichun Feng ${ }^{21}$, Richard Ford ${ }^{57}$, Amélie Fournier ${ }^{44}$, Haonan $\mathrm{Gan}^{32}$, Feng Gao ${ }^{48}$, Alberto Garfagnini ${ }^{61}$, Arsenii Gavrikov ${ }^{61}$, Marco Giammarchi ${ }^{57}$, Agnese Giaz $^{61}$, Nunzio Giudice ${ }^{55}$, Maxim Gonchar ${ }^{67}$, Guanghua Gong ${ }^{13}$, Hui Gong ${ }^{13}$, Yuri Gornushkin ${ }^{67}$, Alexandre Göttel ${ }^{50,48}$, Marco Grassi ${ }^{61}$, Christian Grewing ${ }^{51}$, Vasily Gromov ${ }^{67}$, Minghao $\mathrm{Gu}^{10}$, Xiaofei $\mathrm{Gu}^{37}$, Yu Gu${ }^{19}$, Mengyun Guan ${ }^{10}$, Nunzio Guardone ${ }^{55}$, Maria Gul ${ }^{66}$, Cong Guo ${ }^{10}$, Jingyuan $\mathrm{Guo}^{20}$, Wanlei Guo ${ }^{10}$, Xinheng Guo ${ }^{8}$, Yuhang Guo ${ }^{35,50}$, Paul Hackspacher ${ }^{52}$, Caren Hagner ${ }^{49}$, Ran Han ${ }^{7}$, Yang Han ${ }^{20}$, Muhammad Sohaib Hassan ${ }^{66}$, Miao He ${ }^{10}$, Wei $\mathrm{He}^{10}$, Tobias Heinz ${ }^{54}$, Patrick Hellmuth $^{44}$, Yuekun Heng ${ }^{10}$, Rafael Herrera ${ }^{5}$, YuenKeung Hor $^{20}$, Shaojing Hou ${ }^{10}$, Yee Hsiung ${ }^{40}$, Bei-Zhen $\mathrm{Hu}^{40}$, Hang $\mathrm{Hu}^{20}$, Jianrun $\mathrm{Hu}^{10}$, Jun $\mathrm{Hu}^{10}$, Shouyang $\mathrm{Hu}^{9}$, Tao $\mathrm{Hu}^{10}$, Zhuojun $\mathrm{Hu}^{20}$, Chunhao Huang ${ }^{20}$, Guihong Huang ${ }^{10}$, Hanxiong Huang ${ }^{9}$, Wenhao Huang ${ }^{25}$, Xin Huang ${ }^{10}$, Xingtao Huang ${ }^{25}$, Yongbo Huang ${ }^{28}$, Jiaqi Hui ${ }^{30}$, Lei Huo ${ }^{21}$, Wenju Huo ${ }^{22}$, Cédric Huss ${ }^{44}$, Safeer Hussain $^{66}$, Ara Ioannisian ${ }^{1}$, Roberto Isocrate ${ }^{60}$, Beatrice Jelmini ${ }^{61}$, Kuo-Lun Jen ${ }^{38}$, Ignacio Jeria ${ }^{5}$, Xiaolu $\mathrm{Ji}^{10}$, Xingzhao $\mathrm{Ji}^{20}$, Huihui Jia ${ }^{33}$, Junji $\mathrm{Jia}^{34}$, Siyu Jian ${ }^{9}$, Di Jiang ${ }^{22}$, Wei Jiang ${ }^{10}$, Xiaoshan Jiang $^{10}$, Ruyi Jin ${ }^{10}$, Xiaoping Jing ${ }^{10}$, Cécile Jollet ${ }^{44}$, Jari Joutsenvaara ${ }^{42}$, Sirichok Jungthawan ${ }^{73}$, Leonidas Kalousis ${ }^{45}$, Philipp Kampmann ${ }^{50}$, Li Kang ${ }^{18}$, Rebin Karaparambil ${ }^{47}$, Narine Kazarian ${ }^{1}$, Khanchai Khosonthongkee ${ }^{73}$, Denis Korablev ${ }^{67}$, Konstantin Kouzakov ${ }^{69}$, Alexey Krasnoperov ${ }^{67}$, Andre Kruth ${ }^{51}$, Nikolay Kutovskiy ${ }^{67}$, Pasi Kuusiniemi ${ }^{42}$, Tobias Lachenmaier ${ }^{54}$, Cecilia Landini ${ }^{57}$, Sébastien Leblanc ${ }^{44}$, Victor Lebrin ${ }^{47}$, Frederic Lefevre ${ }^{47}$, Ruiting Lei ${ }^{18}$, Rupert Leitner ${ }^{41}$, Jason Leung ${ }^{38}$, Demin $\mathrm{Li}^{37}$, Fei Li ${ }^{10}$, Fule $\mathrm{Li}^{13}$, Haitao $\mathrm{Li}^{20}$, Huiling $\mathrm{Li}^{10}$, Jiaqi $\mathrm{Li}^{20}$, Mengzhao ${ }^{2}{ }^{10}$, Min $\mathrm{Li}^{11}$, Nan $\mathrm{Li}^{10}$, Nan $\mathrm{Li}^{16}$, Qingjiang $\mathrm{Li}^{16}$, Ruhui $\mathrm{Li}^{10}$, Shanfeng $\mathrm{Li}^{18}$, Tao $\mathrm{Li}^{20}$, Weidong $\mathrm{Li}^{10,14}$, Weiguo $\mathrm{Li}^{10}$, Xiaomei $\mathrm{Li}^{9}$, Xiaonan $\mathrm{Li}^{10}$, Xinglong $\mathrm{Li}^{9}$, $\mathrm{Yi} \mathrm{Li}^{18}$, Yufeng $\mathrm{Li}^{10}$, Zhaohan $\mathrm{Li}^{10}$, Zhib- 
ing $\mathrm{Li}^{20}$, Ziyuan $\mathrm{Li}^{20}$, Hao Liang ${ }^{9}$, Hao Liang ${ }^{22}$, Jiajun Liao ${ }^{20}$, Daniel Liebau ${ }^{51}$, Ayut Limphirat ${ }^{73}$, Sukit Limpijumnong ${ }^{73}$, Guey-Lin $\operatorname{Lin}^{38}$, Shengxin $\operatorname{Lin}^{18}$, Tao Lin ${ }^{10}$, Jiajie Ling ${ }^{20}$, Ivano Lippi ${ }^{60}$, Fang Liu ${ }^{11}$, Haidong $\mathrm{Liu}^{37}$, Hongbang $\mathrm{Liu}^{28}$, Hongjuan $\mathrm{Liu}^{23}$, Hongtao $\mathrm{Liu}^{20}$, Hui Liu ${ }^{19}$, Jianglai $\mathrm{Liu}^{30,31}$, Jinchang Liu ${ }^{10}$, Min Liu ${ }^{23}$, Qian Liu ${ }^{14}$, Qin Liu ${ }^{22}$, Runxuan Liu ${ }^{50,48}$, Shuangyu Liu ${ }^{10}$, Shubin $\mathrm{Liu}^{22}$, Shulin Liu ${ }^{10}$, Xiaowei Liu ${ }^{20}$, Xiwen Liu ${ }^{28}$, Yan Liu ${ }^{10}$, Yunzhe Liu ${ }^{10}$, Alexey Lokhov ${ }^{69,68}$, Paolo Lombardi ${ }^{57}$, Claudio Lombardo ${ }^{55}$, Kai Loo ${ }^{52}$, Chuan Lu ${ }^{32}$, Haoqi Lu ${ }^{10}$, Jingbin Lu ${ }^{15}$, Junguang $\mathrm{Lu}^{10}$, Shuxiang $\mathrm{Lu}^{37}$, Xiaoxu $\mathrm{Lu}^{10}$, Bayarto Lubsandorzhiev ${ }^{68}$, Sultim Lubsandorzhiev ${ }^{68}$, Livia Ludhova ${ }^{50,48}$, Arslan Lukanov ${ }^{68}$, Fengjiao Luo ${ }^{10}$, Guang Luo ${ }^{20}$, Pengwei Luo ${ }^{20}$, Shu Luo ${ }^{36}$, Wuming Luo ${ }^{10}$, Vladimir Lyashuk ${ }^{68}$, Bangzheng $\mathrm{Ma}^{25}$, Qiumei $\mathrm{Ma}^{10}, \mathrm{Si} \mathrm{Ma}^{10}$, Xiaoyan $\mathrm{Ma}^{10}$, Xubo Ma ${ }^{11}$, Jihane Maalmi ${ }^{43}$, Yury Malyshkin ${ }^{67}$, Roberto Carlos Mandujano ${ }^{67}$, Fabio Mantovani ${ }^{56}$, Francesco Manzali ${ }^{61}$, Xin $\mathrm{Mao}^{7}$, Yajun Mao ${ }^{12}$, Stefano M. Mari ${ }^{64}$, Filippo Marini ${ }^{61}$, Sadia Marium $^{66}$, Cristina Martellini ${ }^{64}$, Gisele Martin-Chassard ${ }^{43}$, Agnese Martini ${ }^{63}$, Matthias Mayer ${ }^{53}$, Davit Mayilyan $^{1}$, Ints Mednieks ${ }^{65}$, Yue Meng ${ }^{30}$, Anselmo Meregaglia ${ }^{44}$, Emanuela Meroni ${ }^{57}$, David Meyhöfer $^{49}$, Mauro Mezzetto ${ }^{60}$, Jonathan Miller ${ }^{6}$, Lino Miramonti ${ }^{57}$, Paolo Montini ${ }^{64}$, Michele Montuschi $^{56}$, Axel Müller ${ }^{54}$, Massimiliano Nastasi ${ }^{58}$, Dmitry V. Naumov ${ }^{67}$, Elena Naumova ${ }^{67}$, Diana Navas-Nicolas ${ }^{43}$, Igor Nemchenok ${ }^{67}$, Minh Thuan Nguyen Thi ${ }^{38}$, Feipeng Ning ${ }^{10}$, Zhe Ning ${ }^{10}$, Hiroshi Nunokawa ${ }^{4}$, Lothar Oberauer ${ }^{53}$, Juan Pedro Ochoa-Ricoux ${ }^{74,5}$, Alexander Olshevskiy ${ }^{67}$, Domizia Orestano ${ }^{64}$, Fausto Ortica ${ }^{62}$, Rainer Othegraven ${ }^{52}$, Hsiao-Ru Pan ${ }^{40}$, Alessandro Paoloni ${ }^{63}$, Sergio Parmeggiano ${ }^{57}$, Yatian Pei ${ }^{10}$, Nicomede Pelliccia ${ }^{62}$, Anguo Peng ${ }^{23}$, Haiping Peng ${ }^{22}$, Frédéric Perrot $^{44}$, Pierre-Alexandre Petitjean ${ }^{2}$, Fabrizio Petrucci ${ }^{64}$, Oliver Pilarczyk ${ }^{52}$, Luis Felipe Piñeres Rico $^{45}$, Artyom Popov ${ }^{69}$, Pascal Poussot ${ }^{45}$, Wathan Pratumwan ${ }^{73}$, Ezio Previtali ${ }^{58}$, Fazhi Qi ${ }^{10}$, Ming Qi ${ }^{27}$, Sen Qian ${ }^{10}$, Xiaohui Qian ${ }^{10}$, Zhen Qian ${ }^{20}$, Hao Qiao ${ }^{12}$, Zhonghua Qin ${ }^{10}$, Shoukang Qiu $^{23}$, Muhammad Usman Rajput ${ }^{66}$, Gioacchino Ranucci ${ }^{57}$, Neill Raper ${ }^{20}$, Alessandra Re ${ }^{57}$, Henning Rebber ${ }^{49}$, Abdel Rebii ${ }^{44}$, Bin Ren ${ }^{18}$, Jie Ren ${ }^{9}$, Barbara Ricci ${ }^{56}$, Markus Robens ${ }^{51}$, Mathieu Roche $^{44}$, Narongkiat Rodphai ${ }^{71}$, Aldo Romani ${ }^{62}$, Bedřich Roskovec ${ }^{41}$, Christian Roth ${ }^{51}$, Xiangdong Ruan ${ }^{28}$, Xichao Ruan ${ }^{9}$, Saroj Rujirawat ${ }^{73}$, Arseniy Rybnikov ${ }^{67}$, Andrey Sadovsky ${ }^{67}$, Paolo Saggese $^{57}$, Simone Sanfilippo ${ }^{64}$, Anut Sangka ${ }^{72}$, Nuanwan Sanguansak ${ }^{73}$, Utane Sawangwit ${ }^{72}$, Julia Sawatzki ${ }^{53}$, Fatma Sawy ${ }^{61}$, Michaela Schever ${ }^{50,48}$, Cédric Schwab ${ }^{45}$, Konstantin Schweizer ${ }^{53}$, Alexandr Selyunin ${ }^{67}$, Andrea Serafinii ${ }^{56}$, Giulio Settanta ${ }^{50}$, Mariangela Settimo ${ }^{47}$, Zhuang Shao ${ }^{35}$, Vladislav Sharov ${ }^{67}$, Arina Shaydurova ${ }^{67}$, Jingyan Shi ${ }^{10}$, Yanan Shi ${ }^{10}$, Vitaly Shutov ${ }^{67}$, Andrey Sidorenkov $^{68}$, Fedor Šimkovic ${ }^{70}$, Chiara Sirignano ${ }^{61}$, Jaruchit Siripak ${ }^{73}$, Monica Sisti ${ }^{58}$, Maciej Slupecki $^{42}$, Mikhail Smirnov ${ }^{20}$, Oleg Smirnov ${ }^{67}$, Thiago Sogo-Bezerra ${ }^{47}$, Sergey Sokolov ${ }^{67}$, Julanan Songwadhana ${ }^{73}$, Boonrucksar Soonthornthum ${ }^{72}$, Albert Sotnikov ${ }^{67}$, Ondřej Šrámek ${ }^{41}$, Warintorn Sreethawong ${ }^{73}$, Achim Stahl ${ }^{48}$, Luca Stanco ${ }^{60}$, Konstantin Stankevich ${ }^{69}$, Dušan Štefánik ${ }^{70}$, Hans Steiger ${ }^{52,53}$, Jochen Steinmann ${ }^{48}$, Tobias Sterr ${ }^{54}$, Matthias Raphael Stock ${ }^{53}$, Virginia Strati ${ }^{56}$, Alexander Studenikin ${ }^{69}$, Shifeng Sun ${ }^{11}$, Xilei Sun ${ }^{10}$, Yongjie Sun ${ }^{22}$, Yongzhao Sun ${ }^{10}$, Narumon Suwonjandee ${ }^{71}$, Michal Szelezniak ${ }^{45}$, Jian Tang ${ }^{20}$, Qiang Tang ${ }^{20}$, Quan Tang ${ }^{23}$, Xiao Tang ${ }^{10}$, Alexander Tietzsch ${ }^{54}$, Igor Tkachev ${ }^{68}$, Tomas Tmej ${ }^{41}$, Marco Danilo Claudio Torri ${ }^{41}$, Konstantin Treskov $^{67}$, Andrea Triossi ${ }^{45}$, Giancarlo Troni ${ }^{5}$, Wladyslaw Trzaska ${ }^{42}$, Cristina Tuve ${ }^{55}$, Nikita Ushakov $^{68}$, Johannes van den Boom ${ }^{51}$, Stefan van Waasen ${ }^{51}$, Guillaume Vanroyen ${ }^{47}$, Vadim $\operatorname{Vedin}^{65}$, Giuseppe Verde ${ }^{55}$, Maxim Vialkov ${ }^{69}$, Benoit Viaud ${ }^{47}$, Moritz Vollbrecht ${ }^{50,48}$, Cristina Volpe $^{43}$, Vit Vorobel ${ }^{41}$, Dmitriy Voronin ${ }^{68}$, Lucia Votano ${ }^{63}$, Pablo Walker ${ }^{5}$, Caishen Wang ${ }^{18}$, Chung-Hsiang Wang ${ }^{39}$, En Wang ${ }^{37}$, Guoli Wang ${ }^{21}$, Jian Wang ${ }^{22}$, Jun Wang ${ }^{20}$, Kunyu Wang ${ }^{10}$, 
Lu Wang ${ }^{10}$, Meifen Wang ${ }^{10}$, Meng Wang ${ }^{23}$, Meng Wang ${ }^{25}$, Ruiguang Wang ${ }^{10}$, Siguang Wang ${ }^{12}$, Wei Wang ${ }^{27}$, Wei Wang ${ }^{20}$, Wenshuai Wang ${ }^{10}$, Xi Wang ${ }^{16}$, Xiangyue Wang ${ }^{20}$, Yangfu Wang ${ }^{10}$, Yaoguang Wang ${ }^{10}$, Yi Wang ${ }^{13}$, Yi Wang ${ }^{24}$, Yifang Wang ${ }^{10}$, Yuanqing Wang ${ }^{13}$, Yuman Wang ${ }^{27}$, Zhe Wang ${ }^{13}$, Zheng Wang ${ }^{10}$, Zhimin Wang ${ }^{10}$, Zongyi Wang ${ }^{13}$, Muhammad Waqas ${ }^{66}$, Apimook Watcharangkool ${ }^{72}$, Lianghong Wei ${ }^{10}$, Wei Wei ${ }^{10}$, Wenlu Wei ${ }^{10}$, Yadong Wei ${ }^{18}$, Kaile Wen ${ }^{10}$, Liangjian Wen ${ }^{10}$, Christopher Wiebusch ${ }^{48}$, Steven Chan-Fai Wong ${ }^{20}$, Bjoern Wonsak $^{49}$, Diru Wu $^{10}$, Qun $\mathrm{Wu}^{25}$, Zhi Wu ${ }^{10}$, Michael Wurm ${ }^{52}$, Jacques Wurtz ${ }^{45}$, Christian Wysotzki ${ }^{48}$, Yufei Xi ${ }^{32}$, Dongmei Xia ${ }^{17}$, Xiaochuan $\mathrm{Xie}^{17}$, Yuguang $\mathrm{Xie}^{10}$, Zhangquan $\mathrm{Xie}^{10}$, Zhizhong Xing ${ }^{10}$, Benda $\mathrm{Xu}^{13}$, Cheng $\mathrm{Xu}^{23}$, Donglian $\mathrm{Xu}^{31,30}$, Fanrong $\mathrm{Xu}^{19}$, Hangkun $\mathrm{Xu}^{10}$, Jilei $\mathrm{Xu}^{10}$, Jing $\mathrm{Xu}^{8}$, Meihang $\mathrm{Xu}^{10}$, Yin $\mathrm{Xu}^{33}$, Yu Xu${ }^{50,48}$, Baojun Yan ${ }^{10}$, Taylor Yan ${ }^{73}$, Wenqi Yan ${ }^{10}$, Xiongbo Yan ${ }^{10}$, Yupeng Yan $^{73}$, Anbo Yang ${ }^{10}$, Changgen Yang ${ }^{10}$, Chengfeng Yang ${ }^{28}$, Huan Yang ${ }^{10}$, Jie Yang ${ }^{37}$, Lei Yang ${ }^{18}$, Xiaoyu Yang ${ }^{10}$, Yifan Yang ${ }^{10}$, Yifan Yang ${ }^{2}$, Haifeng Yao ${ }^{10}$, Zafar Yasin ${ }^{66}$, Jiaxuan $\mathrm{Ye}^{10}$, Mei Ye ${ }^{10}$, Ziping $\mathrm{Ye}^{31}$, Ugur Yegin ${ }^{51}$, Frédéric Yermia ${ }^{47}$, Peihuai $\mathrm{Yi}^{10}$, Na Yin ${ }^{25}$, Xiangwei Yin $^{10}$, Zhengyun You ${ }^{20}$, Boxiang $\mathrm{Yu}^{10}$, Chiye $\mathrm{Yu}^{18}$, Chunxu $\mathrm{Yu}^{33}$, Hongzhao $\mathrm{Yu}^{20}$, Miao $\mathrm{Yu}^{34}$, Xianghui $\mathrm{Yu}^{33}$, Zeyuan $\mathrm{Yu}^{10}$, Zezhong Yu ${ }^{10}$, Chengzhuo Yuan ${ }^{10}$, Ying Yuan ${ }^{12}$, Zhenxiong Yuan ${ }^{13}$, Ziyi Yuan ${ }^{34}$, Baobiao Yue ${ }^{20}$, Noman Zafar ${ }^{66}$, Andre Zambanini ${ }^{51}$, Vitalii Zavadskyi ${ }^{67}$, Shan Zeng ${ }^{10}$, Tingxuan Zeng ${ }^{10}$, Yuda Zeng $^{20}$, Liang Zhan ${ }^{10}$, Aiqiang Zhang ${ }^{13}$, Feiyang Zhang ${ }^{30}$, Guoqing Zhang ${ }^{10}$, Haiqiong Zhang ${ }^{10}$, Honghao Zhang ${ }^{20}$, Jiawen Zhang ${ }^{10}$, Jie Zhang ${ }^{10}$, Jin Zhang ${ }^{28}$, Jingbo Zhang ${ }^{21}$, Jinnan Zhang ${ }^{10}$, Peng Zhang ${ }^{10}$, Qingmin Zhang ${ }^{35}$, Shiqi Zhang ${ }^{20}$, Shu Zhang ${ }^{20}$, Tao Zhang ${ }^{30}$, Xiaomei Zhang ${ }^{10}$, Xuantong Zhang ${ }^{10}$, Xueyao Zhang ${ }^{25}$, Yan Zhang ${ }^{10}$, Yinhong Zhang ${ }^{10}$, Yiyu Zhang ${ }^{10}$, Yongpeng Zhang $^{10}$, Yuanyuan Zhang ${ }^{30}$, Yumei Zhang ${ }^{20}$, Zhenyu Zhang ${ }^{34}$, Zhijian Zhang ${ }^{18}$, Fengyi Zhao ${ }^{26}$, Jie Zhao ${ }^{10}$, Rong Zhao ${ }^{20}$, Shujun Zhao ${ }^{37}$, Tianchi Zhao ${ }^{10}$, Dongqin Zheng ${ }^{19}$, Hua Zheng ${ }^{18}$, Minshan Zheng ${ }^{9}$, Yangheng Zheng ${ }^{14}$, Weirong Zhong ${ }^{19}$, Jing Zhou ${ }^{9}$, Li Zhou ${ }^{10}$, Nan Zhou ${ }^{22}$, Shun Zhou $^{10}$, Tong Zhou ${ }^{10}$, Xiang Zhou ${ }^{34}$, Jiang Zhu ${ }^{20}$, Kangfu Zhu ${ }^{35}$, Kejun Zhu ${ }^{10}$, Zhihang Zhu ${ }^{10}$, Bo Zhuang ${ }^{10}$, Honglin Zhuang ${ }^{10}$, Liang Zong ${ }^{13}$ and Jiaheng Zou ${ }^{10}$

${ }^{1}$ Yerevan Physics Institute, Yerevan, Armenia. ${ }^{2}$ Université Libre de Bruxelles, Brussels, Belgium. ${ }^{3}$ Universidade Estadual de Londrina, Londrina, Brazil. ${ }^{4}$ Pontificia Universidade Catolica do Rio de Janeiro, Rio, Brazil. ${ }^{5}$ Pontificia Universidad Católica de Chile, Santiago, Chile. ${ }^{6}$ Universidad Tecnica Federico Santa Maria, Valparaiso, Chile. ${ }^{7}$ Beijing Institute of Spacecraft Environment Engineering, Beijing, China. ${ }^{8}$ Beijing Normal University, Beijing, China. ${ }^{9}$ China Institute of Atomic Energy, Beijing, China. ${ }^{10}$ Institute of High Energy Physics, Beijing, China. ${ }^{11}$ North China Electric Power University, Beijing, China. ${ }^{12}$ School of Physics, Peking University, Beijing, China. ${ }^{13}$ Tsinghua University, Beijing, China. ${ }^{14}$ University of Chinese Academy of Sciences, Beijing, China. ${ }^{15}$ Jilin University, Changchun, China. ${ }^{16}$ College of Electronic Science and Engineering, National University of Defense Technology, Changsha, China. ${ }^{17}$ Chongqing University, Chongqing, China. ${ }^{18}$ Dongguan University of Technology, Dongguan, China. ${ }^{19}$ Jinan University, Guangzhou, China. ${ }^{20}$ Sun Yat-Sen University, Guangzhou, China. ${ }^{21}$ Harbin Institute of Technology, Harbin, China. ${ }^{22}$ University of Science and Technology of China, Hefei, China. ${ }^{23}$ The Radiochemistry and Nuclear Chemistry Group in University of South China, Hengyang, China. ${ }^{24}$ Wuyi University, Jiangmen, China. ${ }^{25}$ Shandong University, Jinan, China, and Key Laboratory of Particle Physics and Particle Irradiation of Ministry of Education, Shandong University, Qingdao, China. ${ }^{26}$ Institute of Modern Physics, Chinese Academy of Sciences, Lanzhou, China. ${ }^{27}$ Nanjing University, Nanjing, China. ${ }^{28}$ Guangxi University, Nanning, China. ${ }^{29}$ East China University of Science and Technology, 
Shanghai, China. ${ }^{30}$ School of Physics and Astronomy, Shanghai Jiao Tong University, Shanghai, China. ${ }^{31}$ Tsung-Dao Lee Institute, Shanghai Jiao Tong University, Shanghai, China. ${ }^{32}$ Institute of Hydrogeology and Environmental Geology, Chinese Academy of Geological Sciences, Shijiazhuang, China. ${ }^{33}$ Nankai University, Tianjin, China. ${ }^{34}$ Wuhan University, Wuhan, China. ${ }^{35} \mathrm{Xi}$ 'an Jiaotong University, Xi'an, China. ${ }^{36}$ Xiamen University, Xiamen, China. ${ }^{37}$ School of Physics and Microelectronics, Zhengzhou University, Zhengzhou, China. ${ }^{38}$ Institute of Physics, National Yang Ming Chiao Tung University, Hsinchu. ${ }^{39}$ National United University, Miao-Li. ${ }^{40}$ Department of Physics, National Taiwan University, Taipei. ${ }^{41}$ Charles University, Faculty of Mathematics and Physics, Prague, Czech Republic. ${ }^{42}$ University of Jyvaskyla, Department of Physics, Jyvaskyla, Finland. ${ }^{43}$ IJCLab, Université Paris-Saclay, CNRS/IN2P3, 91405 Orsay, France. ${ }^{44}$ Univ. Bordeaux, CNRS, CENBG, UMR 5797, F-33170 Gradignan, France. ${ }^{45}$ IPHC, Université de Strasbourg, CNRS/IN2P3, F-67037 Strasbourg, France. ${ }^{46}$ Centre de Physique des Particules de Marseille, Marseille, France. ${ }^{47}$ SUBATECH, Université de Nantes, IMT Atlantique, CNRS-IN2P3, Nantes, France. ${ }^{48}$ III. Physikalisches Institut B, RWTH Aachen University, Aachen, Germany. ${ }^{49}$ Institute of Experimental Physics, University of Hamburg, Hamburg, Germany. ${ }^{50}$ Forschungszentrum Jülich $\mathrm{GmbH}$, Nuclear Physics Institute IKP-2, Jülich, Germany. ${ }^{51}$ Forschungszentrum Jülich $\mathrm{GmbH}$, Central Institute of Engineering, Electronics and Analytics - Electronic Systems (ZEA-2), Jülich, Germany. ${ }^{52}$ Institute of Physics, Johannes-Gutenberg Universität Mainz, Mainz, Germany. ${ }^{53}$ Technische Universität München, München, Germany. ${ }^{54}$ Eberhard Karls Universität Tübingen, Physikalisches Institut, Tübingen, Germany. ${ }^{55}$ INFN Catania and Dipartimento di Fisica e Astronomia dell Università di Catania, Catania, Italy. ${ }^{56}$ Department of Physics and Earth Science, University of Ferrara and INFN Sezione di Ferrara, Ferrara, Italy. ${ }^{57}$ INFN Sezione di Milano and Dipartimento di Fisica dell Università di Milano, Milano, Italy. ${ }^{58}$ INFN Milano Bicocca and University of Milano Bicocca, Milano, Italy. ${ }^{59}$ INFN Milano Bicocca and Politecnico of Milano, Milano, Italy. ${ }^{60}$ INFN Sezione di Padova, Padova, Italy. ${ }^{61}$ Dipartimento di Fisica e Astronomia dell'Università di Padova and INFN Sezione di Padova, Padova, Italy. ${ }^{62}$ INFN Sezione di Perugia and Dipartimento di Chimica, Biologia e Biotecnologie dell'Università di Perugia, Perugia, Italy. ${ }^{63}$ Laboratori Nazionali di Frascati dell'INFN, Roma, Italy. ${ }^{64}$ University of Roma Tre and INFN Sezione Roma Tre, Roma, Italy. ${ }^{65}$ Institute of Electronics and Computer Science, Riga, Latvia. ${ }^{66}$ Pakistan Institute of Nuclear Science and Technology, Islamabad, Pakistan. ${ }^{67}$ Joint Institute for Nuclear Research, Dubna, Russia. ${ }^{68}$ Institute for Nuclear Research of the Russian Academy of Sciences, Moscow, Russia. ${ }^{69}$ Lomonosov Moscow State University, Moscow, Russia. ${ }^{70}$ Comenius University Bratislava, Faculty of Mathematics, Physics and Informatics, Bratislava, Slovakia. ${ }^{71}$ Department of Physics, Faculty of Science, Chulalongkorn University, Bangkok, Thailand. ${ }^{72}$ National Astronomical Research Institute of Thailand, Chiang Mai, Thailand. ${ }^{73}$ Suranaree University of Technology, Nakhon Ratchasima, Thailand. ${ }^{74}$ Department of Physics and Astronomy, University of California, Irvine, California, USA. 